OPEN ACCESS

Edited by:

Michal Masternak, University of Central Florida,

United States

Reviewed by:

Vinal Menon,

University of Minnesota Twin Cities,

United States

John S. Ji,

Duke Kunshan University, China

*Correspondence:

Yao He

yhe301@x263.net

Shanshan Yang

shanqinhua001@163.com

${ }^{t}$ These authors share first authorship

Specialty section:

This article was submitted to

Endocrinology of Aging,

a section of the journal

Frontiers in Endocrinology

Received: 18 February 2021 Accepted: 07 April 2021

Published: 29 June 2021

Citation:

Han K, Jia W, Wang S, Cao W,

Song $Y$, Wang J, Liu M, Yang $S$ and He Y (2021) Synergistic Impact of Body Mass Index and Cognitive

Function on All-Cause Mortality

in Older Adults: A Nationwide

Longitudinal Study.

Front. Endocrinol. 12:620261.

doi: 10.3389/fendo.2021.620261

\section{Synergistic Impact of Body Mass Index and Cognitive Function on All-Cause Mortality in Older Adults: A Nationwide Longitudinal Study}

\author{
Ke Han ${ }^{1,2 t}$, Wangping Jia ${ }^{1,2 t}$, Shengshu Wang ${ }^{2}$, Wenzhe Cao ${ }^{1,2}$, Yang Song ${ }^{1,2}$, \\ Jianwei Wang ${ }^{1,2}$, Miao $\mathrm{Liu}^{3}$, Shanshan Yang ${ }^{4 *}$ and Yao $\mathrm{He}^{2 *}$
}

${ }^{1}$ Medical School of Chinese PLA, Beijing, China, ${ }^{2}$ Institute of Geriatrics, Beijing Key Laboratory of Aging and Geriatrics, National Clinical Research Center for Geriatrics Diseases, State Key Laboratory of Kidney Disease, The 2nd Medical Center of Chinese PLA General Hospital, Beijing, China, ${ }^{3}$ Department of Statistics and Epidemiology, Medical School of Chinese PLA, Beijing, China, ${ }^{4}$ Department of Disease Prevention and Control, The 1st Medical Center, Chinese PLA General Hospital, Beijing, China

Background: Body mass index (BMI) and cognitive function are independent predictors of mortality risk. However, little is known about the combined impact of BMl and cognitive function on the risk of all-cause mortality in older adults. In this study, we aimed to examine the associations between BMI, cognitive function, and all-cause mortality, including between-factor interactions, in the general population of older adults in China.

Methods: We used the data between 2011 and 2018 from the Chinese Longitudinal Healthy Longevity Survey that included adults aged $\geq 65$ years residing in the 23 provinces of China. The association between BMl and cognitive function on all-cause mortality was examined with the Cox proportional hazards regression model.

Results: The study included 8,293 Chinese older adults. Low BMI (underweight) and cognitive impairment were associated with the highest risk of death after adjustments [hazard ratio $(\mathrm{HR})=2.18$; 95\% confidence interval $(\mathrm{Cl}), 1.96-2.41]$; this combined effect was more prominent among adults aged $<100$ years and women. In addition, there was an interaction effect of BMl and cognitive impairment on all-cause mortality $(P<0.001)$. Concurrently, among older adults with normal cognition, the risk of mortality related to underweight was higher than among their cognitively impaired counterparts [55\% (normal cognition) vs. 38\% (cognitive impairment)].

Conclusions: Low BMI (underweight) and cognitive impairment were independently and jointly associated with increased risk of all-cause mortality among Chinese older adults, and females showed a stronger effect in this association. The association between BMI and mortality was more pronounced in the participants with normal cognition than in their cognitively impaired counterparts.

Keywords: cognition, body mass index, malnutrition, older adults, mortality 


\section{INTRODUCTION}

Body mass index (BMI) has been previously investigated in several studies dedicated to older adults and is considered a predictor of premature mortality in this population. BMI has been reported to follow a U-shaped $(1,2)$ or J-shaped $(3-5)$ association with mortality, and the impact of obesity on mortality risk may decrease with age (6) and over time (7). However, accruing evidence demonstrates that for communitydwelling older adults, low BMI may have a more pronounced impact on mortality risk than high $\operatorname{BMI}(8,9)$. Compared to Western populations, the Chinese population has lower BMI and a higher proportion of underweight individuals (10), suggesting that the impact of low BMI on outcomes, including mortality, in the Chinese older adults may be distinct and warrants further investigation.

Cognitive impairment (CI) is a major public health concern because it is associated with adverse economic and sociopsychological outcomes (11). In aging societies, the prevalence of CI is gradually increasing, in particular, among older adults (12), and the number of people affected is expected to exceed 140 million by 2050 (13). China has one of the fastest aging populations worldwide (14), which translates to a high economic burden associated with CI (15). CI contributes toward a loss of capacity for self-care, reduced quality of life, and increased risk of disability and premature mortality $(16,17)$.

There is a close association between BMI and cognitive function in older adults. Previous studies of older adults across various ethnic groups revealed that being underweight is associated with an increased risk of cognitive decline (18-20) and that BMI and cognitive function share a genetic basis (21, 22). Although these factors are important contributors to the survival and well-being of older adults, their combined effects on the risk of mortality remain unclear and require further investigation. Hence, in this study, we aimed to examine the independent and synergistic impact of BMI and CI on the risk of all-cause mortality using data from the Chinese Longitudinal Healthy Longevity Survey (CLHLS), conducted on a nationally representative sample of older adults in China.

\section{METHODS}

\section{Study Setting and Participants}

We used the 2011-2018 longitudinal data of the CLHLS, a nationwide, prospective, longitudinal study of Chinese community-dwelling older adults. The CLHLS used a multistage, stratified cluster sampling, and recruited older Chinese adults from half the cities or counties in 23 out of the 31 provinces of mainland China, constituting $85 \%$ of the Chinese population. A detailed description of sampling frame and investigation methods of CLHLS has been outlined in previous reports (23).

Participants in this study were recruited from the sixth wave (2011/2012) of CLHLS. Among the 9765 participants enrolled in the baseline interview, we included all older adults aged 65 or above (excluding 86 participants with age mismatch) and with
BMI information and cognitive assessment (excluding 530 participants with missing data) at baseline. After exclusion of 856 subjects lost to follow-up, a total of 8293 older adults were included for the analysis. Figure $\mathbf{S 1}$ shows the full process of the inclusion and exclusion of the participants.

Ethical approval was granted by the Ethics Committee of Peking University and Duke University. Written informed consent was obtained from all individual participants included in the study.

\section{Data Collection \\ BMI}

Weight and height were measured during the survey. Body mass index was calculated as bodyweight $(\mathrm{kg})$ divided by squared body height $\left(\mathrm{m}^{2}\right)$. According to the recommended classifications for Chinese adults (24), we define underweight as BMI $<18.5 \mathrm{~kg} / \mathrm{m}^{2}$, normal as $18.5-23.9 \mathrm{~kg} / \mathrm{m}^{2}$, overweight as $24-27.9 \mathrm{~kg} / \mathrm{m}^{2}$, and obesity as $\geq 28 \mathrm{~kg} / \mathrm{m}^{2}$.

\section{Cognitive Function}

Cognitive performance was assessed by the Chinese version of Mini-Mental State Examination (MMSE), which is adapted from the scale developed by Folstein and colleagues (25). It measures cognitive function domains including orientation, recognition, attention, memory and language. All questions were answered by the respondent in person during the face-to-face interview. About $2 \%$ of the interviewees were "unable to answer" some of the questions due to low cognitive function as the interviewer indicated (26). Therefore, we encoded these answers as incorrect answers (scored 0 ) during data processing. The total score ranges from 0 to 30, with higher scores indicating better cognitive functions (27). Cognitive impairment (CI) was defined using education-based cutoff points $(28,29):<18$, participants with no formal education; $<24$, participants with 1-6 years of education; $<25$, participants with more than 6 years of education; those who did not to meet these criteria were defined as having normal cognition.

\section{All-Cause Mortality}

For the respondents who died before the next wave of surveys, a specific questionnaire was used to obtain comprehensive death information. Survival status and date of death were collected from official death certificates when available. Otherwise, the information was collected from close relatives who were familiar with the deceased or the local residents committee. Survival time was calculated from the date of the baseline interview to the date of death (for participants who died) or the interview date of the last follow-up survey (for participants who were alive). Those who cannot be contacted were assigned a "lost follow-up" status and were censored in the analysis.

\section{Covariates}

Covariate information were collected from the structured questionnaire. Demographic information included age (continuous), sex (men or women), ethnicity (Han or others), residence (city, town or rural), and education ( $\geq 1$ year of schooling or $<1$ year of schooling). We also considered 
lifestyle characteristics such as smoking status (current, former or never), alcohol drinking status (current, former or never), and weekly exercise (current, former or never) and self-reported medical history including hypertension, diabetes mellitus, cardiovascular disease, stroke and cerebrovascular disease, respiratory disease and cancer. Impairment in activities of Daily Living Disorder (ADL) was defined as a participant being dependent in at least one aspect of using the toilet, bathing, indoor activities, dressing, eating, or continence. Whether the older adults suffered from spinal deformity was judged by the interviewer based on observation rather than direct inquiry in order to avoid offending the participants. In addition, quality of sleep (five levels from "very good" to "very bad") and access to adequate medical service (yes vs. no) were collected.

\section{Statistical Analyses}

In all analyses, we categorized the BMI and cognition into binary variables and created a 4-level joint variable including: $\mathrm{BMI} \geq 18.5$ $\mathrm{kg} / \mathrm{m}^{2}$ and normal cognition, $\mathrm{BMI}<18.5 \mathrm{~kg} / \mathrm{m}^{2}$ and normal cognition, $\mathrm{BMI} \geq 18.5 \mathrm{~kg} / \mathrm{m}^{2}$ and $\mathrm{CI}$, and $\mathrm{BMI}<18.5 \mathrm{~kg} / \mathrm{m}^{2}$ and CI.

Baseline characteristic of all participants were represented by the 4 groups. Continuous variables were expressed as mean and standard deviation (SD) and categorized variables were expressed as number and percentage. The differences between the means and proportions of the two groups were compared by analysis of variance (ANOVA) and chi-square test. We evaluated all-cause mortality by the 4-level joint variable of BMI/CI with Kaplan-Meier survival plots. Equality of survival rates was tested with log-rank tests.

We conducted multivariable Cox proportional hazard models to calculate the hazard ratios (HRs) for all-cause mortality by the four groups. The basic model was adjusted for age, sex, ethnicity, residence, education; and the fully adjusted model additionally adjusted for smoking, alcohol drinking, weekly exercise, ADL impairment, spinal deformity, hypertension, diabetes mellitus, cardiovascular disease, stroke and cerebrovascular disease, respiratory disease and cancer. The significance of multiplicative interaction between BMI levels and CI for allcause mortality was tested by adding cross-product terms in the models. Based on the fully adjusted Cox model, we used restricted cubic splines with knots at $18.5 \mathrm{~kg} / \mathrm{m}^{2}$ (BMI) and 24 points (MMSE) (30) to flexibly model the association of BMI and cognition performance with all-cause mortality. In this part, we also observed the association between BMI and all-cause mortality by CI categories.

In order to further observe the influence of important confounding factors such as age and sex in this association, we conducted subgroup analyses based on the fully adjusted Cox model above. We also tested the interactions between the 4-level joint variable of $\mathrm{BMI} / \mathrm{CI}$ and subgroup factors for allcause mortality.

We performed several sensitivity analyses to evaluated the robustness of our results: 1) additionally adjusting for medical treatment accessibility as the deprivation of medical treatment may increase the mortality risk (31);2) additionally adjusting for sleep quality which is an important factor related to BMI (32), CI (33) and all-cause mortality (34); 3) excluding the participants with spinal deformity; 4) excluding the participants whose BMI > $24 \mathrm{~kg} / \mathrm{m}^{2}$; and 5) excluding the participants who died in the first year. All statistical analyses were performed using $\mathrm{R}$ (version 3.6.3) and Empower Stats (X\&Y Solutions, Inc., Boston, MA). A p-value $<0.05$ (2-tailed) was considered statistically significant.

\section{RESULTS}

Table 1 presents the baseline characteristics of 8,293 (4,555 women) participants included in this study, by the joint variable of BMI and CI. Participants' mean age was 85.53 (SD, 11.07) years, ranging between 65 and 104 years. The average BMI was $21.20(\mathrm{SD}, 4.23) \mathrm{kg} / \mathrm{m}^{2}$ and the average MMSE score was 22.77 (SD, 8.82). Among all participants, 2,164 (764 men and 1,400 women) older adults were underweight, accounting for $26.09 \%$ of the sample. In addition, 1,882 (553 men and 1,329 women) older adults had CI, accounting for $22.70 \%$ of the sample. Participants who had both CI and low BMI (underweight) were more likely to be older, female, having a lower level of education, having lower ADL scores, and having no history of smoking or alcohol use, or a weekly exercise habit than their counterparts. There were differences in the prevalence of spinal deformity, hypertension, diabetes mellitus, cardiovascular diseases, stroke and cerebrovascular diseases, respiratory diseases and dementia between the groups. The Kaplan-Meier survival curves revealed distinct outcome trajectories for the groups (logrank p-value $<0.001$, Figure 1). The median survival time was $54.93,28.16$, and 21.75 months for the groups with normal cognition/underweight, CI/normal weight/obesity, and CI/ underweight, respectively.

During the 7-year follow-up period, 856 participants were lost to follow-up (drop-out rate, 9.36\%). The participants lost to follow-up were older and less likely to regularly consume alcohol or have a spinal deformity, had lower MMSE scores, towndwelling, had lower levels of education, and a higher prevalence of cerebrovascular diseases and stroke (see Table S1) than their retained counterparts.

\section{BMI and Cognitive Function, and All-Cause Mortality}

In total, 4,212 (1,836 men and 2,376 women) participants died during an unweighted median follow-up period of 40.44 (interquartile range, 25.53-79.24) months. Restricted cubic splines flexibly modeled and visualized the independent associations between BMI and cognitive function, and all-cause mortality (Figures 2A, B). There was a non-linear association between BMI and the MMSE score, and all-cause mortality in all participants (all P for non-linear <0.001). Low BMI was consistently associated with increased risk of all-cause mortality, but further increase of BMI values had a marginal impact on the risk of all-cause mortality. Compared to the reference point, as the MMSE score increases from 0 to 30, the risk of all-cause mortality continues to decrease.

Table 2 presents the combined association between BMI and $\mathrm{CI}$, and all-cause mortality. The normal cognition and $\mathrm{BMI} \geq 18.5$ $\mathrm{kg} / \mathrm{m}^{2}$ group was used as the reference. In the unadjusted model, 
TABLE 1 | Baseline characteristics of participants by BMl and cognition categories.

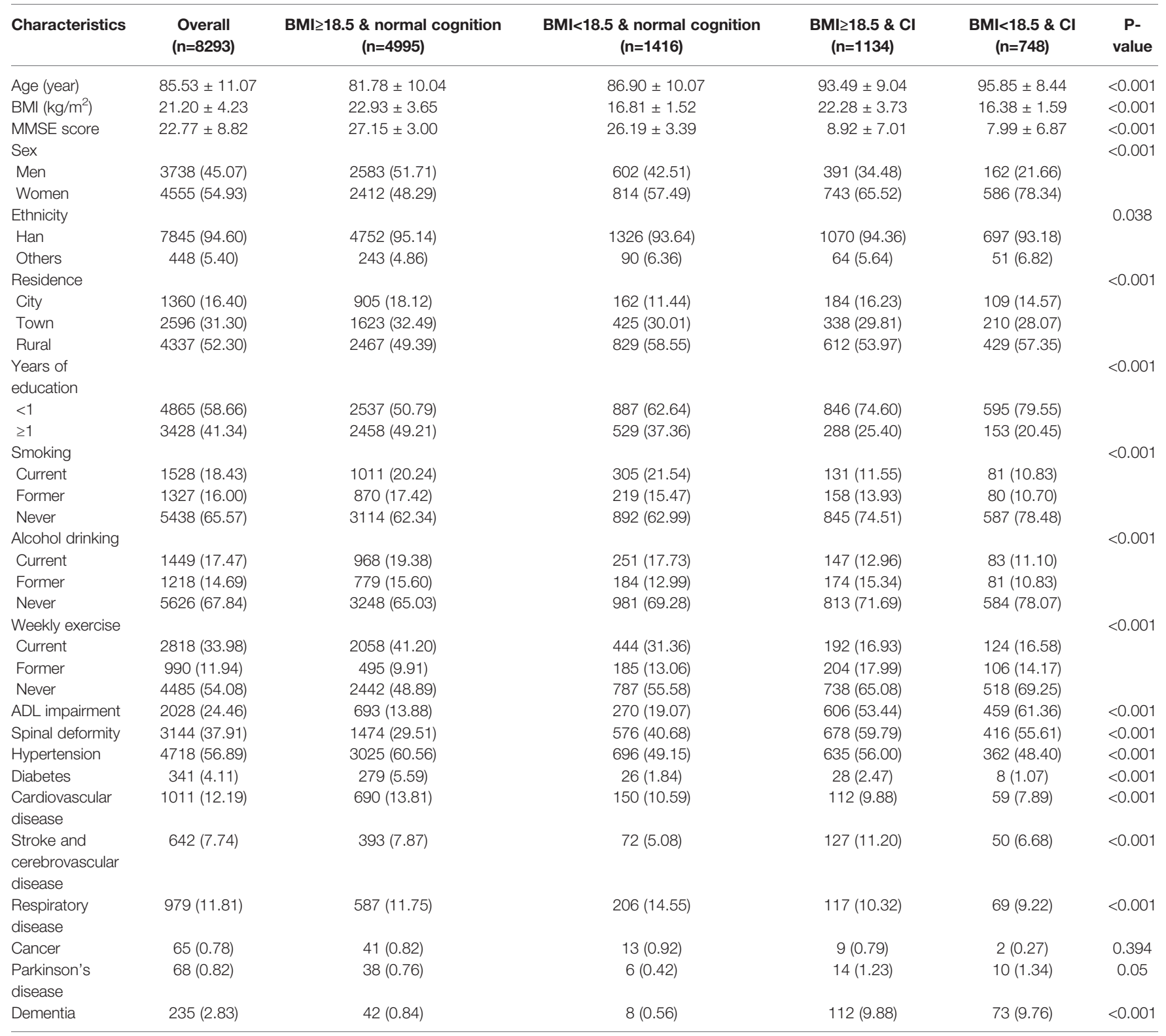

Data were reported as the mean \pm standard deviation $(S D)$ for continuous variables and number (\%) for categorized variables.

Differences between groups were evaluated by analysis of variance (ANOVA) or chi-square test.

$B M I$, body mass index; $C l$, cognitive impairment; $A D L$, activity of daily living.

CI $[\mathrm{HR}=1.94,95 \%$ confidence interval (CI), 1.79-2.11], low BMI (underweight) $(\mathrm{HR}=3.88,95 \% \mathrm{CI}, 3.58-4.20)$, and the combination of these factors $(\mathrm{HR}=5.00,95 \% \mathrm{CI}, 4.57-5.48)$ were associated with a higher risk of all-cause mortality. In the adjusted model, compared with the reference group, CI, low BMI (underweight), and the combination of these factors yielded the following effect estimates: $\mathrm{HR}=1.55$ (95\% CI, 1.42-1.68), 1.80 (95\% CI, 1.64-1.96), and 2.18 (95\% CI, 1.96-2.41), respectively.

There was a significant interaction effect of BMI and CI on all-cause mortality in all models (p-values for interaction < 0.001). Compared to other groups, the combination of lower BMI (underweight) and CI was associated with the highest risk of all-cause mortality in the fully adjusted model. The impact of
BMI on the risk of mortality was the higher among people with normal cognition than that among cognitively impaired participants [55\% (normal cognition) vs. 38\% (CI)]. In an analysis stratified by cognitive function categories, restricted cubic splines for remained a reverse-J shape and we found that the all-cause mortality risk varied flatter in participants with normal cognition (Figure 2C). The difference was more significant in women and participants aged $<100$ years than in men or centenarians (Figure S2).

\section{Subgroup and Sensitivity Analyses}

Subgroup analyses revealed that the combined impact of BMI and $\mathrm{CI}$ on mortality risk was more prominent among adults aged 


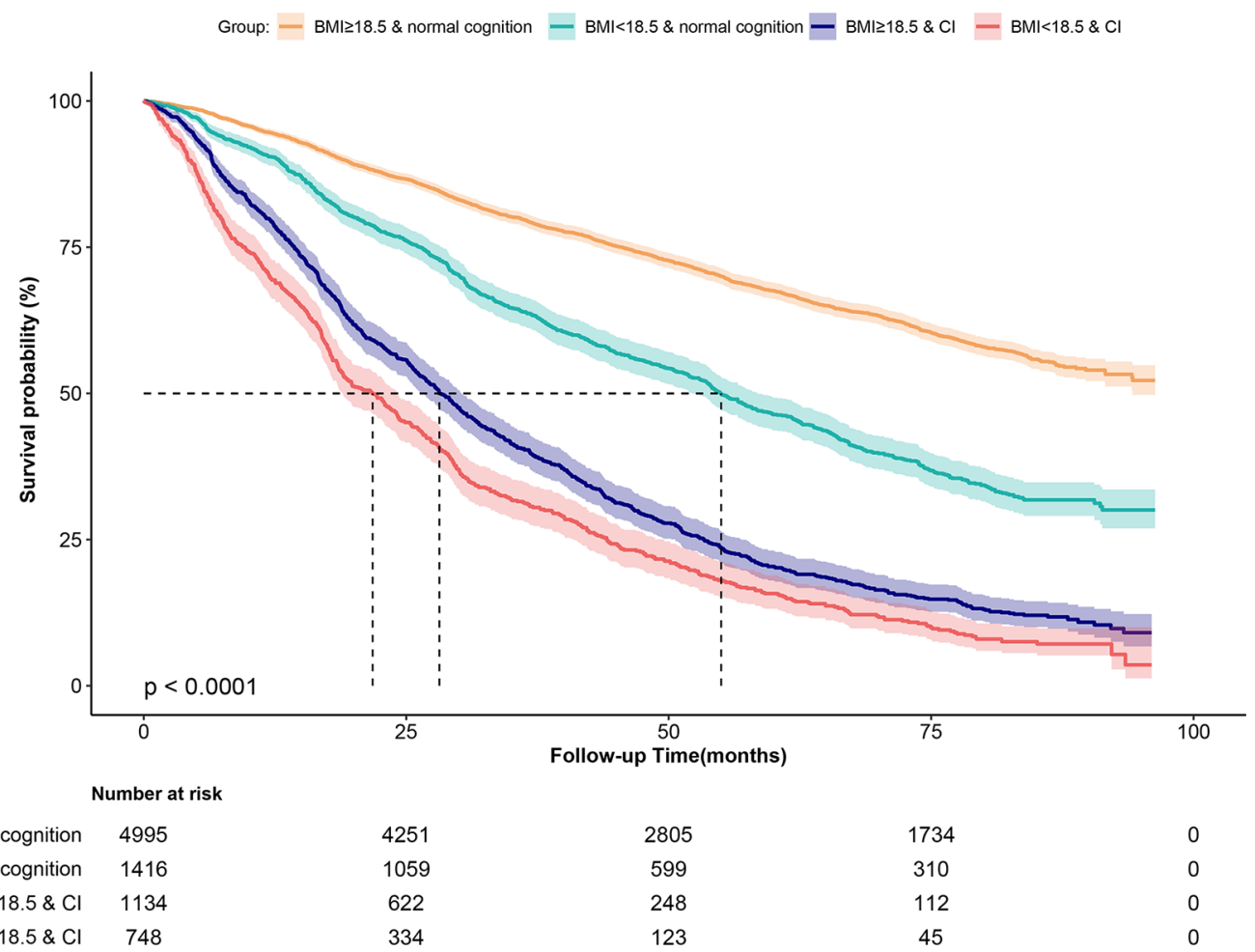

FIGURE 1 | Kaplan-Meier survival curves for Chinese older adults stratified by the 4-level joint BMI/cognition groups.

$<100$ years and women than among centenarians or men (Figure 3). We found the associations between the joint variable of $\mathrm{BMI} / \mathrm{CI}$ and mortality by age and sex showed significant interaction effects $(\mathrm{P}=0.156$ and 0.026 , respectively). In the group aged $>100$ years, the differences between estimated effect sizes associated with different BMI categories [32\% (normal cognition) vs. 38\% (CI)] were inconsistent with the estimates of the same parameters for the overall sample; however, the interaction effect of BMI and CI on all-cause mortality in centenarians was not significant $(\mathrm{P}$ for interaction $=0.640$ ). In particular, we found in the group younger than 80 years that when underweight, CI existed alone or both existed,

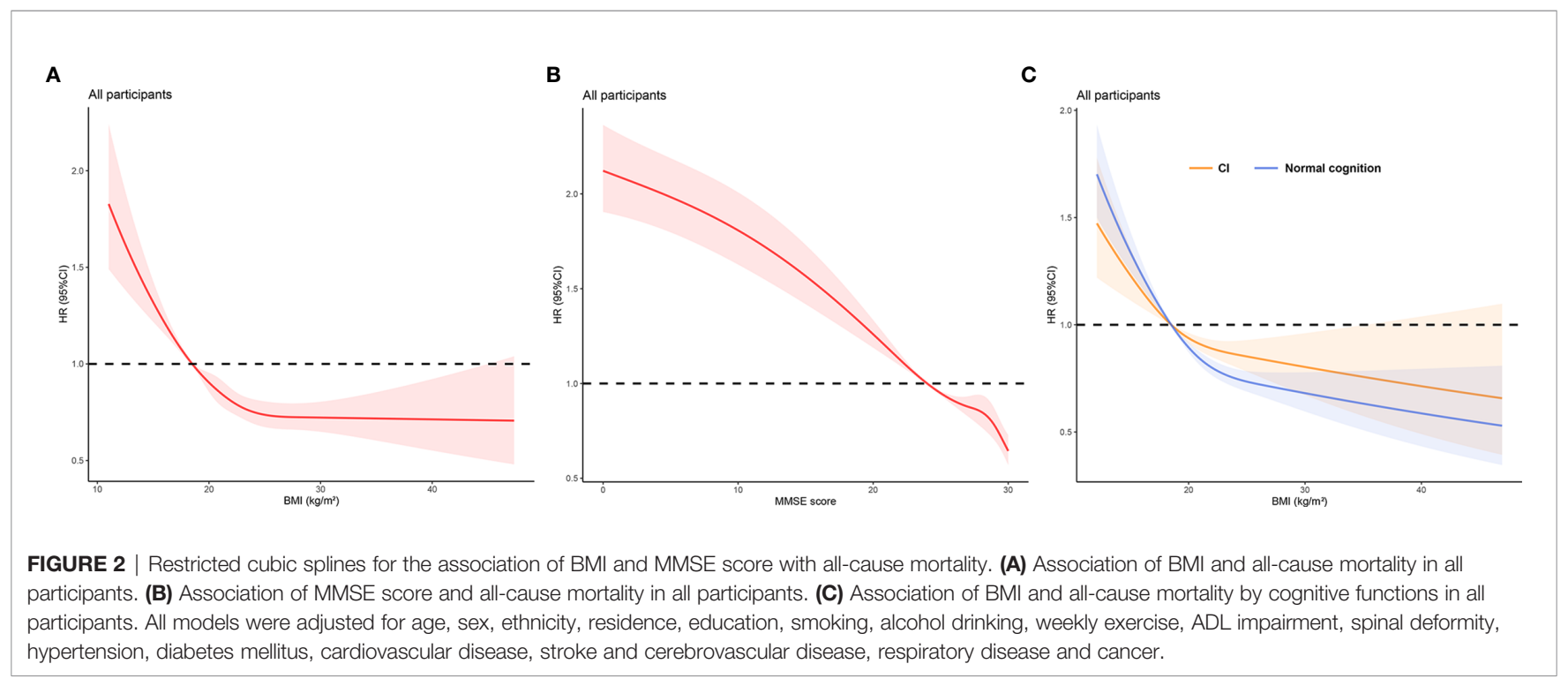


TABLE 2 | Hazard ratios for the combined associations of BMl and cognitive impairment with all-cause mortality.

\begin{tabular}{|c|c|c|c|c|}
\hline & \multirow[t]{2}{*}{ No. of deaths } & \multicolumn{3}{|c|}{ HRs for all-cause mortality } \\
\hline & & Unadjusted model & Basic model $^{a}$ & Fully adjusted model ${ }^{b}$ \\
\hline \multicolumn{5}{|l|}{ Normal cognition } \\
\hline $\mathrm{BMI} \geq 18.5 \mathrm{~kg} / \mathrm{m}^{2}$ & 1825 & Ref. & Ref. & Ref. \\
\hline $\mathrm{BMl}<18.5 \mathrm{~kg} / \mathrm{m}^{2}$ & 827 & $1.94(1.79,2.11)$ & $1.52(1.39,1.65)$ & $1.55(1.42,1.68)$ \\
\hline \multicolumn{5}{|l|}{$\mathrm{Cl}$} \\
\hline $\mathrm{BMl} \geq 18.5 \mathrm{~kg} / \mathrm{m}^{2}$ & 917 & $3.88(3.58,4.20)$ & $2.10(1.93,2.29)$ & $1.80(1.64,1.96)$ \\
\hline $\mathrm{BMl}<18.5 \mathrm{~kg} / \mathrm{m}^{2}$ & 643 & $5.00(4.57,5.48)$ & $2.48(2.25,2.74)$ & $2.18(1.96,2.41)$ \\
\hline P for interaction & - & $<0.001$ & $<0.001$ & $<0.001$ \\
\hline
\end{tabular}

HR, hazard ratio; BMI, body mass index; $\mathrm{Cl}$, cognitive impairment.

${ }^{a}$ Basic model: adjusted for age, sex, ethnicity, residence, education.

${ }^{b}$ Fully adjusted model: additionally adjusted for smoking, alcohol drinking, weekly exercise, ADL impairment, spinal deformity, hypertension, diabetes mellitus, cardiovascular disease, stroke and cerebrovascular disease, respiratory disease and cancer.

the association with the risk of all-cause mortality was the strongest in all age groups, but no significant interaction effect was found in this group (P for interaction $=0.300$, Table S2).

After additional adjustments for access to medical services and quality of sleep, the combined impact of BMI and CI on allcause mortality remained similar to that estimated in the primary analysis (Table S3). However, the combined impact of BMI and CI on all-cause mortality became more pronounced after participants with spinal deformities were excluded. Exclusion of the participants who were overweight or obese (BMI $>24 \mathrm{~kg}$ / $\left.\mathrm{m}^{2}\right)$ at baseline $(\mathrm{n}=1,728)$ did not affect the effect estimates. Exclusion of the participants who died in the first year $(n=860)$ slightly decreased the magnitude of the effect estimates. All models in sensitivity analyses demonstrated the stability of interaction effects between binary BMI categories and CI on the risk of all-cause mortality.

\section{DISCUSSION}

In this large prospective community-based study of 8,293 Chinese older adults, we observed a reverse J-shaped association between BMI and all-cause mortality and a positive association between cognitive function and all-cause mortality. Compared to the participants with normal cognition and normal or excess weight, the participants who were both underweight and affected by CI had the highest risk of mortality after adjustment for potential confounders; this combined effect was more prominent among older adults aged $<100$ years and women than among centenarians or men. It should be noted that the association between BMI and mortality risk varied between older adults, depending on their cognitive function. To the best of our knowledge, this is the first study report the synergistic effects of BMI and cognitive function on the risk of all-cause mortality in older adults.
A

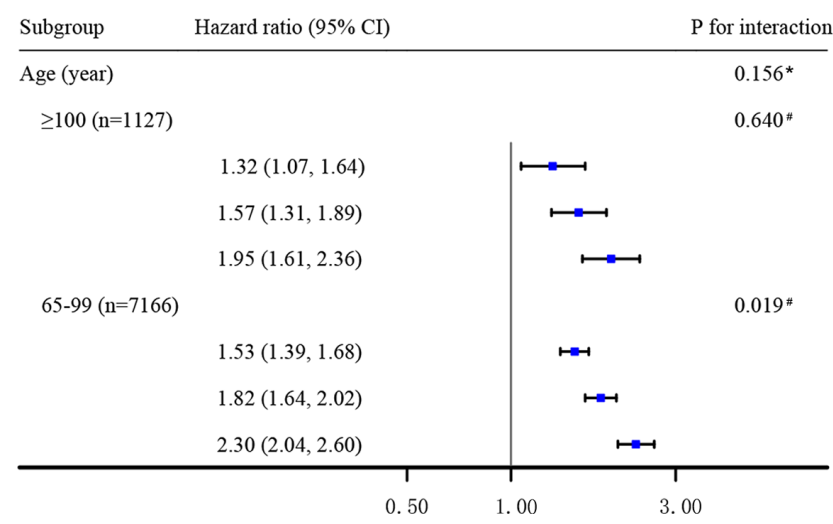

B

\begin{tabular}{|c|c|c|}
\hline Subgroup & Hazard ratio $(95 \% \mathrm{CI})$ & P for interaction \\
\hline Sex & & $0.026^{*}$ \\
\hline Female & & $0.004^{\#}$ \\
\hline
\end{tabular}

$1.55(1.38,1.74)$

$2.04(1.81,2.29)$

$2.48(2.18,2.81)$

Male (n=3738)

$1.56(1.38,1.77)$

$1.49(1.30,1.72)$

$1.68(1.39,2.04)$

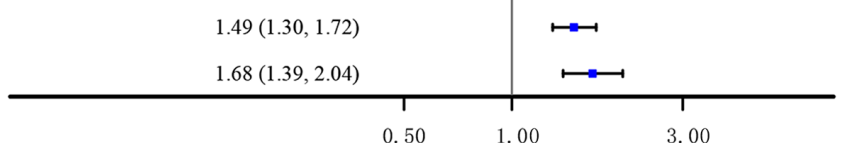

FIGURE 3 | Hazard ratios for the combined associations of BMl and Cl with all-cause mortality according to the classification of age (A) and sex (B). All models were adjusted for age, sex (not in Panel B), ethnicity, residence, education, smoking, alcohol drinking, weekly exercise, ADL impairment, spinal deformity, hypertension, diabetes mellitus, cardiovascular disease, stroke and cerebrovascular disease, respiratory disease and cancer. The estimated HRs for each subgroup are compared with the $\mathrm{BMl} \geq 18.5 \mathrm{~kg} / \mathrm{m}^{2}$ and normal cognition group (not shown) and presented in the order of $\mathrm{BMl}<18.5 \mathrm{~kg} / \mathrm{m}^{2}$ and normal cognition group, $\mathrm{BMl} \geq 18.5 \mathrm{~kg} / \mathrm{m}^{2}$ and $\mathrm{Cl}$ group, and $\mathrm{BMl}<18.5 \mathrm{~kg} / \mathrm{m}^{2}$ and $\mathrm{Cl}$ group, respectively. ${ }^{*}$ Interaction between the 4-level joint variable of $\mathrm{BMI} / \mathrm{Cl}$ and age or sex subgroup on all-cause mortality. " Interaction between BMl levels and cognitive impairment on all-cause mortality. 
In this study, $26.09 \%$ of the participants were underweight, which is a much higher proportion than that previously reported in other large cohort studies $(6,7)$. Previous studies have reported on the independent association between BMI and allcause mortality risk in older adults $(2,35,36)$. A previous metaanalysis of studies focused on adults aged $\geq 65$ years demonstrated a U-shaped association between BMI and mortality, whereby the lowest risk of death was detected among individuals with BMI between $24.0 \mathrm{~kg} / \mathrm{m}^{2}$ and $30.9 \mathrm{~kg}$ / $\mathrm{m}^{2}$; in fact, no association was detected between high BMI (overweight) and increased risk of mortality (8). However, this meta-analysis included studies conducted in Europe, North America, Australia, and Canada. In China, studies on the population of adults aged $\geq 65$ years $(9,37,38)$ reported findings similar to those of our study, whereby high BMI (overweight/obesity) was not associated with increased risk of all-cause mortality. We are not certain that this finding supports the obesity paradox. Evidence has shown that the morbidity and mortality of various chronic diseases in obese people increase significantly. Compared with the normal weight, overweight/ obese individuals tend to die earlier, thus survivor bias might exist in the older population in this study. In addition, the prevalence of obesity in the Chinese population is limited to the last 30 years, and it affects people with higher socioeconomic status more (9), who have easier access to better medical and health service. It may cover up the real association between high BMI and all-cause mortality. Furthermore, in contrast to high BMI, low BMI (underweight) had a significant association with all-cause mortality in this study. Evidence from a large cohort study has shown that weight loss might have a greater impact on the risk of mortality than weight gain in people aged $>60$ years (39). These findings may update policy development around health promotion and disease prevention among older adults in China.

Several previous studies have explored interaction effects of BMI and age (40), low-grade inflammation (41), and cardiovascular disease categories (42) on mortality risk in different populations. Our study expanded on this evidence, showing a significant interaction of BMI and cognitive function on all-cause mortality. Nevertheless, the present finding is inconsistent with that of a previous study, which did not find any interaction between BMI and the MMSE scores (43). However, this previous study included participants aged $\geq 70$ years, residing in northern Italy; the study sample size was also relatively small.

The mechanism underlying the association between BMI, cognitive function, and all-cause mortality remains unclear. One potential explanation may be that low BMI (underweight) and CI may be surrogate indicators of underlying disease rather than direct contributors to mortality risk. In a study of 2,550 Chinese older adults aged $\geq 55$ years, the prevalence of poor cognitive function was relatively high among people with low BMI and chronic comorbidity; meanwhile, low BMI was significantly associated with poor cognitive performance only in individuals with pre-existing chronic comorbidities (44). In addition, phenotypic and genetic associations have been reported between BMI and cognitive function, suggesting shared genetic basis and common biological pathways (22). The direction of phenotypic correlation indicated that lower BMI was associated with better cognitive function, which suggests that the relationship between BMI, cognitive function, and mortality risk is complex and likely mediated by several other factors.

A previous review has shown that the association between BMI and cognition, and mortality risk varies between life stages (45). Specifically, in the moderately old aged 75-84, high BMI (overweight and obesity) is associated with an increased risk of future dementia; however, in later life, this association changes, and higher BMI and better cognitive function begin to correspond to reduced mortality risk, in particular, when examining executive functions and language performance (46). This finding might help explain the discrepancy in findings between the centenarians included in the present study and the relatively younger adults; however, confirming this association requires further research.

Finally, higher body fat mass, which is closely related to higher BMI, has been reported to positively correlate with cognitive performance (47). Due to prominent exposure to endogenous estrogen, this association was more pronounced in older women than in men $(48,49)$. This finding might account for the differences in the present study findings between men and women.

The present findings are based on data from the long-term longitudinal study of community-dwelling general adult population in China. Low BMI (underweight), in particular, when concurrent with CI was significantly associated with increased all-cause mortality risk during the 7-year follow-up period. Based on this evidence, we suggest that appropriate anthropometric measurements and cognitive screening should be included in the health and care plan of older adults in China. Furthermore, in the present study, older adults with normal cognition experienced a stronger effect of low BMI (underweight) on the risk of mortality than their counterparts with CI. This finding suggests that maintaining optimal BMI is paramount also among older adults with normal cognitive function. Nevertheless, proactive assessment and improvement of cognitive function, as required, among individuals with low BMI (underweight) may help reduce their risk of premature mortality to a greater extent than a nutritional intervention alone.

The present study has several limitations. First, this study accounted for BMI only; no other anthropometric parameters such as body fat mass or distribution were considered. This limitation might have affected our ability to propose a mechanism underlying the presented associations, warranting further studies. Meanwhile, using a single indicator might have weakened our effect estimates because of the presence of regression dilution (50). However, excluding subjects with BMI $>24 \mathrm{~kg} / \mathrm{m}^{2}$ marginally affected the results, suggesting the validity of the presented estimates. Second, the present study relied on self-reported medical history, resulting in estimates of chronic diseases that were lower than those reported for the general older adult population in China. Therefore, the impact of chronic 
disease on the presented estimates (including confounding, interaction, and mediating effects) needs to be examined (44). Third, this study did not differentiate between causes of death; as a result, the presented mortality risks are composite estimates that preclude conclusions regarding specific cardiovascular or metabolic disease-associated risks that would be required to inform heath policies in these areas.

\section{CONCLUSIONS}

In conclusion, the present study demonstrates that the general older adult population in China concurrently affected by low BMI (underweight) and CI is at the highest risk of premature allcause mortality; within that group, women and older adults aged $<100$ years are at particularly high risk. Among the participants with normal cognition, the association between BMI and allcause mortality was more prominent than among the participants with CI. These results suggest that cognitive screening is required for designing precise and effective interventions in this group. Further studies are required to validate these findings.

\section{DATA AVAILABILITY STATEMENT}

The datasets presented in this study can be found in online repositories. The names of the repository/repositories and accession number(s) can be found below: https:/www.icpsr. umich.edu/icpsrweb/NACDA/series/487.

\section{ETHICS STATEMENT}

The studies involving human participants were reviewed and approved by the Ethics Committee of Peking University and Duke University. The patients/participants provided their written informed consent to participate in this study. Written

\section{REFERENCES}

1. Adams KF, Schatzkin A, Harris TB, Kipnis V, Mouw T, Ballard-Barbash R, et al. Overweight, Obesity, and Mortality in a Large Prospective Cohort of Persons 50 to 71 Years Old. N Engl J Med (2006) 355(8):763-78. doi: 10.1056/NEJMoa055643

2. Cheng FW, Gao X, Mitchell DC, Wood C, Still CD, Rolston D, et al. Body Mass Index and All-Cause Mortality Among Older Adults. Obesity (Silver Spring) (2016) 24(10):2232-9. doi: 10.1002/oby.21612

3. Calle EE, Thun MJ, Petrelli JM, Rodriguez C, Heath CWJr. Body-Mass Index and Mortality in a Prospective Cohort of U.S. Adults. N Engl J Med (1999) 341 (15):1097-105. doi: 10.1056/nejm199910073411501

4. Sun YQ, Burgess S, Staley JR, Wood AM, Bell S, Kaptoge SK, et al. Body Mass Index and All Cause Mortality in HUNT and UK Biobank Studies: Linear and non-Linear Mendelian Randomisation Analyses. Bmj (2019) 364:11042. doi: 10.1136/bmj.11042

5. Jee SH, Sull JW, Park J, Lee SY, Ohrr H, Guallar E, et al. Body-Mass Index and Mortality in Korean Men and Women. N Engl J Med (2006) 355(8):779-87. doi: 10.1056/NEJMoa054017 informed consent was obtained from the individual(s) for the publication of any potentially identifiable images or data included in this article.

\section{AUTHOR CONTRIBUTIONS}

The authors' responsibilities were as follows- $\mathrm{KH}, \mathrm{WJ}, \mathrm{SW}, \mathrm{WC}$, SY, YS, JW, ML, and YH: designed the research. WC, YS, and JW: collected the data. $\mathrm{KH}, \mathrm{WJ}$, and SW: performed the statistical analysis. $\mathrm{KH}$ : wrote the paper. $\mathrm{KH}, \mathrm{WJ}$, and $\mathrm{YH}$ : had primary responsibility for the final content. All authors contributed to the article and approved the submitted version.

\section{FUNDING}

The study was supported by Opening Foundation of State Key Laboratory of Kidney Diseases (KF-01-115), the National Natural Science Foundation of China (81773502, 81703285 and 81703308), Beijing Nova Program (Z181100006218085), the Medical Big Data Fund of Chinese PLA General Hospital (2018MBD-029) and the Opening Foundation of National Clinical Research Center of Geriatrics (NCRCGPLAGH-2017017).

\section{ACKNOWLEDGMENTS}

We appreciate all the staff of the CLHLS for their continued cooperation and contribution to the field work.

\section{SUPPLEMENTARY MATERIAL}

The Supplementary Material for this article can be found online at: https://www.frontiersin.org/articles/10.3389/fendo.2021.620261/ full\#supplementary-material

6. Bhaskaran K, Dos-Santos-Silva I, Leon DA, Douglas IJ, Smeeth L. Association of BMI With Overall and Cause-Specific Mortality: A Population-Based Cohort Study of 3.6 Million Adults in the UK. Lancet Diabetes Endocrinol (2018) 6(12):944-53. doi: 10.1016/s2213-8587(18) 30288-2

7. Flegal KM, Graubard BI, Williamson DF, Gail MH. Excess Deaths Associated With Underweight, Overweight, and Obesity. Jama (2005) 293(15):1861-7. doi: 10.1001/jama.293.15.1861

8. Winter JE, MacInnis RJ, Wattanapenpaiboon N, Nowson CA. BMI and AllCause Mortality in Older Adults: A Meta-Analysis. Am J Clin Nutr (2014) 99 (4):875-90. doi: 10.3945/ajcn.113.068122

9. Wang J, Taylor AW, Zhang T, Appleton S, Shi Z. Association Between Body Mass Index and All-Cause Mortality Among Oldest Old Chinese. J Nutr Health Aging (2018) 22: (2):262-8. doi: 10.1007/s12603-017-0907-2

10. World Health Organization. Obesity: Preventing and Managing the Global Epidemic. Report of a WHO Consultation. World Health Organ Tech Rep Ser (2000) 894:i-xii, 1-253. 
11. Bassuk SS, Wypij D, Berkman LF. Cognitive Impairment and Mortality in the Community-Dwelling Elderly. Am J Epidemiol (2000) 151(7):676-88. doi: 10.1093/oxfordjournals.aje.a010262

12. Sosa-Ortiz AL, Acosta-Castillo I, Prince MJ. Epidemiology of Dementias and Alzheimer's Disease. Arch Med Res (2012) 43(8):600-8. doi: 10.1016/ j.arcmed.2012.11.003

13. Prince M, Wimo A, Guerchet M, Ali G-M, Wu YT, Prina M, et al. World Alzheimer Report 2015. London: Alzheimer's Disease International (ADI (2015). Available at: https://www.alz.co.uk/research/WorldAlzheimer Report2015.pdf.

14. Nations U. World Population Ageing 2017. United Nations (2017), Contract No.: ST/ESA/SER.A/408.

15. Jia JP, Wei CB, Chen SQ, Li FY, Tang Y, Qin W, et al. The Cost of Alzheimer's Disease in China and Re-Estimation of Costs Worldwide. Alzheimers Dement (2018) 14: (4):483-91. doi: 10.1016/j.jalz.2017.12.006

16. Shimada H, Makizako H, Lee S, Doi T, Lee S, Tsutsumimoto K, et al. Impact of Cognitive Frailty on Daily Activities in Older Persons. J Nutr Health Aging (2016) 20: (7):729-35. doi: 10.1007/s12603-016-0685-2

17. Zeng Y, Feng QS, Hesketh T, Christensen K, Vaupel JW. Survival, Disabilities in Activities of Daily Living, and Physical and Cognitive Functioning Among the Oldest-Old in China: A Cohort Study. Lancet (2017) 389(10079):1619-29. doi: 10.1016/s0140-6736(17)30548-2

18. Estrella-Castillo DF, Gómez-de-Regil L. Comparison of Body Mass Index Range Criteria and Their Association With Cognition, Functioning and Depression: A Cross-Sectional Study in Mexican Older Adults. BMC Geriatr (2019) 19(1):339. doi: 10.1186/s12877-019-1363-0

19. Arvanitakis Z, Capuano AW, Bennett DA, Barnes LL. Body Mass Index and Decline in Cognitive Function in Older Black and White Persons. J Gerontol A Biol Sci Med Sci (2018) 73(2):198-203. doi: 10.1093/gerona/glx152

20. Kim S, Kim Y, Park SM. Body Mass Index and Decline of Cognitive Function. PloS One (2016) 11(2):e0148908-e. doi: 10.1371/journal.pone.0148908

21. Frazier-Wood AC, Carnell S, Pena O, Hughes SO, O’Connor TM, Asherson P, et al. Cognitive Performance and BMI in Childhood: Shared Genetic Influences Between Reaction Time But Not Response Inhibition. Obesity (Silver Spring) (2014) 22(11):2312-8. doi: 10.1002/oby.20862

22. Marioni RE, Yang J, Dykiert D, Mõttus R, Campbell A, Davies G, et al. Assessing the Genetic Overlap Between BMI and Cognitive Function. Mol Psychiatry (2016) 21(10):1477-82. doi: 10.1038/mp.2015.205

23. Zeng Y. Towards Deeper Research and Better Policy for Healthy Aging Using the Unique Data of Chinese Longitudinal Healthy Longevity Survey. China Economic J (2012) 5(2-3):131-49. doi: 10.1080/17538963.2013.764677

24. Zhou BF. Predictive Values of Body Mass Index and Waist Circumference for Risk Factors of Certain Related Diseases in Chinese Adults-Study on Optimal Cut-Off Points of Body Mass Index and Waist Circumference in Chinese Adults. BioMed Environ Sci (2002) 15(1):83-96. doi: 10.1046/j.14406047.11.s8.9.x

25. Folstein MF, Folstein SE, McHugh PR. "Mini-Mental State". A Practical Method for Grading the Cognitive State of Patients for the Clinician. J Psychiatr Res (1975) 12(3):189-98. doi: 10.1016/0022-3956(75)90026-6

26. Zhang Z, Gu D, Hayward MD. Childhood Nutritional Deprivation and Cognitive Impairment Among Older Chinese People. Soc Sci Med (2010) 71: (5):941-9. doi: 10.1016/j.socscimed.2010.05.013

27. Zhang Z. Gender Differentials in Cognitive Impairment and Decline of the Oldest Old in China. J Gerontol B Psychol Sci Soc Sci (2006) 61(2):S107-15. doi: 10.1093/geronb/61.2.s107

28. Zhang MY, Katzman R, Salmon D, Jin H, Cai GJ, Wang ZY, et al. The Prevalence of Dementia and Alzheimer's Disease in Shanghai, China: Impact of Age, Gender, and Education. Ann Neurol (1990) 27(4):428-37. doi: 10.1002/ana.410270412

29. Crum RM, Anthony JC, Bassett SS, Folstein MF. Population-Based Norms for the Mini-Mental State Examination by Age and Educational Level. Jama (1993) 269(18):2386-91. doi: 10.1001/jama.269.18.2386

30. Katzman R, Zhang MY, Ouang Ya Q, Wang ZY, Liu WT, Yu E, et al. A Chinese Version of the Mini-Mental State Examination; Impact of Illiteracy in a Shanghai Dementia Survey. J Clin Epidemiol (1988) 41(10):971-8. doi: 10.1016/0895-4356(88)90034-0

31. Bechini A, Pieralli F, Chellini E, Martini A, Dugheri G, Crescioli F, et al. Application of Socio-Economic-Health Deprivation Index, Analysis of
Mortality and Influenza Vaccination Coverage in the Elderly Population of Tuscany. J Prev Med Hyg (2018) 59(4 Suppl 2):E18-e25. doi: 10.15167/24214248/jpmh2018.59.4s2.1116

32. Wu IHC, Nguyen N, Balachandran DD, Lu Q, McNeill LH. Sleep and Obesity: The Mediating Role of Health Behaviors Among African Americans. Sleep Health (2019) 5(2):193-200. doi: 10.1016/j.sleh.2018.12.005

33. Ma XQ, Jiang CQ, Xu L, Zhang WS, Zhu F, Jin YL, et al. Sleep Quality and Cognitive Impairment in Older Chinese: Guangzhou Biobank Cohort Study. Age Ageing (2019) 49(1):119-24. doi: 10.1093/ageing/afz120

34. Morgan K, Hartescu I. Sleep Duration and All-Cause Mortality: Links to Physical Activity and Prefrailty in a 27-Year Follow Up of Older Adults in the UK. Sleep Med (2019) 54:231-7. doi: 10.1016/j.sleep.2018.11.008

35. Veronese N, Cereda E, Solmi M, Fowler SA, Manzato E, Maggi S, et al. Inverse Relationship Between Body Mass Index and Mortality in Older Nursing Home Residents: A Meta-Analysis of 19,538 Elderly Subjects. Obes Rev (2015) 16(11):1001-15. doi: 10.1111/obr.12309

36. An R, Liu GG. Cognitive Impairment and Mortality Among the Oldest-Old Chinese. Int J Geriatr Psychiatry (2016) 31(12):1345-53. doi: 10.1002/ gps. 4442

37. Huang C, Elo IT. Mortality of the Oldest Old Chinese: The Role of Early-Life Nutritional Status, Socio-Economic Conditions, and Sibling SexComposition. Popul Stud (Camb) (2009) 63(1):7-20. doi: 10.1080/ 00324720802626921

38. Sun H, Ren X, Chen Z, Li C, Chen S, Wu S, et al. Association Between Body Mass Index and Mortality in a Prospective Cohort of Chinese Adults. Medicine (Baltimore) (2016) 95(32):e4327. doi: 10.1097/md. 0000000000004327

39. Kim YH, Kim SM, Han KD, Son JW, Lee SS, Oh SW, et al. Change in Weight and Body Mass Index Associated With All-Cause Mortality in Korea: A Nationwide Longitudinal Study. J Clin Endocrinol Metab (2017) 102 (11):4041-50. doi: 10.1210/jc.2017-00787

40. Palvannan P, Miranda I, Merchant AM. The Combined Effect of Age and Body Mass Index on Outcomes in Foregut Surgery: A Regression Model Analysis of the National Surgical Quality Improvement Program Data. Surg Endosc (2016) 30(6):2572-82. doi: 10.1007/s00464-015-4529-7

41. Crotti G, Gianfagna F, Bonaccio M, Di Castelnuovo A, Costanzo S, Persichillo $\mathrm{M}$, et al. Body Mass Index and Mortality in Elderly Subjects From the MoliSani Study: A Possible Mediation by Low-Grade Inflammation? Immunol Invest (2018) 47(8):774-89. doi: 10.1080/08820139.2018.1538237

42. Lamelas P, Schwalm JD, Leong D, Jolly S, Mehta S, Bangdiwala S, et al. Varying Effects of Body Mass Index and Mortality in Different Risk Groups. Am J Cardiol (2018) 122(7):1155-60. doi: 10.1016/j.amjcard.2018.06.038

43. Gustafson DR, Mazzuco S, Ongaro F, Antuono P, Forloni G, Albani D, et al. Body Mass Index, Cognition, Disability, APOE Genotype, and Mortality: The "Treviso Longeva" Study. Am J Geriatr Psychiatry (2012) 20(7):594-602. doi: 10.1097/JGP.0b013e31823031a4

44. Ng TP, Feng L, Niti M, Yap KB. Albumin, haemoglobin, BMI and cognitive performance in older adults. Age Ageing (2008) 37(4):423-9. doi: 10.1093/ ageing/afn102

45. García-Ptacek S, Faxén-Irving G, Cermáková P, Eriksdotter M, Religa D. Body Mass Index in Dementia. Eur J Clin Nutr (2014) 68(11):1204-9. doi: 10.1038/ ejcn.2014.199

46. Schmeidler J, Mastrogiacomo CN, Beeri MS, Rosendorff C, Silverman JM. Distinct Age-Related Associations for Body Mass Index and Cognition in Cognitively Healthy Very Old Veterans. Int Psychogeriatr (2019) 31(6):895-9. doi: $10.1017 / \mathrm{s} 1041610218001412$

47. Tikhonoff V, Casiglia E, Guidotti F, Giordano N, Martini B, Mazza A, et al. Body Fat and the Cognitive Pattern: A Population-Based Study. Obesity (Silver Spring) (2015) 23(7):1502-10. doi: 10.1002/oby.21114

48. Bagger YZ, Tankó LB, Alexandersen P, Qin G, Christiansen C. The Implications of Body Fat Mass and Fat Distribution for Cognitive Function in Elderly Women. Obes Res (2004) 12(9):1519-26. doi: 10.1038/oby.2004.189

49. Noh HM, Oh S, Song HJ, Lee EY, Jeong JY, Ryu OH, et al. Relationships Between Cognitive Function and Body Composition Among CommunityDwelling Older Adults: A Cross-Sectional Study. BMC Geriatr (2017) 17 (1):259. doi: 10.1186/s12877-017-0651-9

50. Emberson JR, Whincup PH, Morris RW, Walker M, Lowe GD, Rumley A. Extent of Regression Dilution for Established and Novel Coronary Risk 
Factors: Results From the British Regional Heart Study. Eur J Cardiovasc Prev Rehabil (2004) 11(2):125-34. doi: 10.1097/01.hjr.0000114967. 39211.e5

Conflict of Interest: The authors declare that the research was conducted in the absence of any commercial or financial relationships that could be construed as a potential conflict of interest.
Copyright (c) 2021 Han, Jia, Wang, Cao, Song, Wang, Liu, Yang and He. This is an open-access article distributed under the terms of the Creative Commons Attribution License (CC BY). The use, distribution or reproduction in other forums is permitted, provided the original author(s) and the copyright owner(s) are credited and that the original publication in this journal is cited, in accordance with accepted academic practice. No use, distribution or reproduction is permitted which does not comply with these terms. 